\title{
A NOTE ON CONTACT SURGERY DIAGRAMS
}

\author{
BURAK OZBAGCI
}

ABSTRACT. We prove that for any positive integer $k$ the stabilization of a $\frac{1}{k}$-surgery curve in a contact surgery diagram induces an overtwisted contact structure.

\section{INTRODUCTION}

In [DG], Ding and Geiges proved that every (closed) contact 3-manifold can be given by a contact $( \pm 1)$-surgery on a Legendrian link in the standard contact $S^{3}$. Here we use the parenthesis to emphasize that the surgery coefficients are measured with respect to the contact framing. If the coefficients of all the curves in a contact surgery diagram are $(-1)$, then it is well-known that the contact structure is Stein fillable and hence tight. It is possible, however, that a contact surgery diagram without any $(-1)$-surgery curve induces a tight contact structure. Consider for example the contact structure given by the surgery diagram in Figure 1. Topologically this is equivalent to the 0 -surgery on the unknot in $S^{3}$, hence the diagram in Figure 1 induces a contact structure on $S^{1} \times S^{2}$. In fact, this diagram represents the unique (up to isotopy) tight contact structure on $S^{1} \times S^{2}$. The tightness of this contact structure can be proven using the convex surface theory (cf. [DGS1]) or the contact Ozsváth-Szabó invariants (cf. [LS]). We will give a simple argument (cf. [Sti]) to prove that this contact structure is Stein fillable (hence tight) in Section 5.

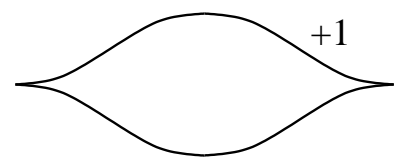

FiguRE 1. The unique tight contact structure on $S^{1} \times S^{2}$

Let $k$ be a positive integer. In this note we show that if a contact surgery diagram contains a $\frac{1}{k}$-surgery curve which has an isolated stabilized arc then the induced contact structure is overtwisted ${ }^{1}$. By stabilizing a Legendrian knot we mean the addition of an extra "zig-zag" as depicted in Figure 2. By an isolated arc of a surgery

\footnotetext{
${ }^{1} \mathrm{An}$ alternative proof of this result was pointed out to us by John Etnyre. He can explicitly construct the overtwisted disk using different methods.
} 
curve in a contact surgery diagram we mean a connected arc of a component of the Legendrian surgery link which does not have any crossings with the rest of the link as illustrated also in Figure 2. Notice that a contact $\frac{1}{k}$-surgery on a Legendrian knot is uniquely defined as opposed to a general rational contact surgery. In fact, performing a contact $\frac{1}{k}$-surgery on a Legendrian knot $K$ is equivalent to performing a contact (+1)-surgery on each one of the $k$ Legendrian knots $K_{1}, K_{2}, \cdots, K_{k}$ which are obtained by successive Legendrian push-offs of $K$ (cf. [DG], [DGS1]).

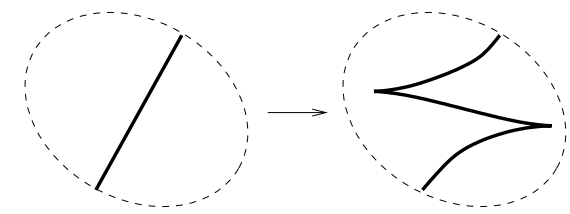

(a)

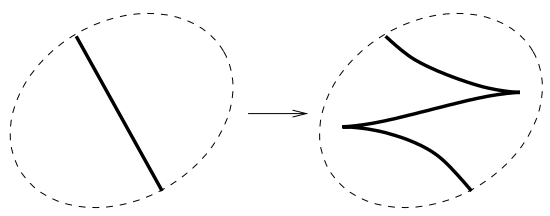

(b)

FiguRE 2. Stabilizing an isolated arc of a Legendrian knot

In other words, we prove that any contact surgery diagram which contains a stabilized $\frac{1}{k}$-curve, where the stabilized arc is not entangled with any other part of the Legendrian link, is overtwisted. Notice that by stabilizing a surgery curve we not only change the contact structure but also the 3-manifold carrying the contact structure. For example the contact structure on $S^{3}$ induced by the surgery diagram depicted in Figure 3 (which is a stabilization of the unknot in Figure 1) is overtwisted. This was proved in [DGS1] by producing the overtwisted disk in the surgered 3-manifold. The same argument can be used to prove the overtwistedness of the contact structure obtained by performing a contact $(+1)$-surgery on the stabilization of any Legendrian knot (cf. [DGS2]). Our result is a generalization of theirs in the case of a contact $\frac{1}{k}$-surgery (for all $k>0$ ), although we use a different method.

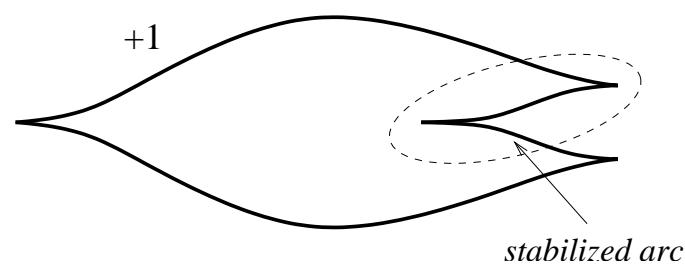

FiguRE 3. An overtwisted contact structure on $S^{3}$

It is clear that our generalization holds true only under the assumption that the stabilization takes place in an isolated arc of the surgery curve. If the stabilized arc of the $\frac{1}{k}$-surgery curve can not be isolated then the contact structure induced by the 
surgery may be tight. For a canonical example consider a surgery diagram which consists of a $(+1)$-surgery on an arbitrary Legendrian knot and a ( -1$)$-surgery on its Legendrian push-off. Then by $[\mathrm{DG}]$ the resulting contact structure is isotopic to the standard tight contact structure on $S^{3}$. This is illustrated in Figure 4.

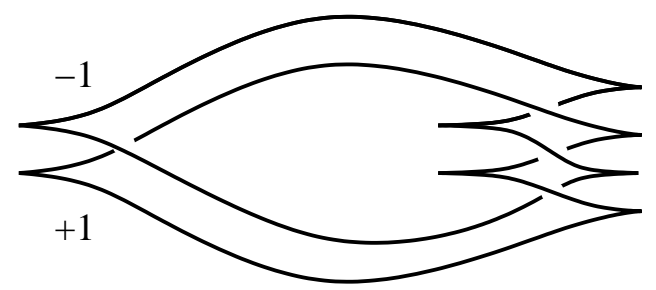

Figure 4. Cancelling a contact $(+1)$-surgery

On the other hand consider the Legendrian knot in Figure 3 with its Legendrian push-off and apply contact $(+1)$-surgery to both curves (cf. Figure 5). We will prove that the resulting contact structure is overtwisted.

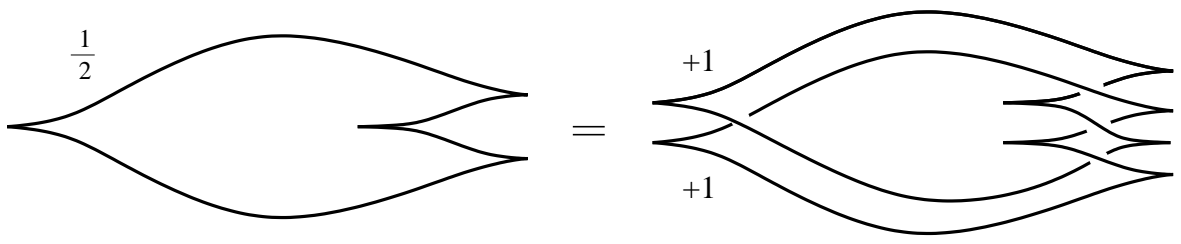

FiguRE 5. An overtwisted contact structure

Although our main result does not seem to have an immediate application, it partially answers the curious question of to what extend we can use $(+1)$-surgery curves in a contact surgery diagram to guarantee the overtwistedness of the induced contact structure in the light of the fact that the lack of $(+1)$-curves guarantees Stein fillability and hence tightness of the given contact structure. Of course there are examples (cf. [LS]) of tight but non-fillable (in any sense) contact structures induced by contact $(+1)$-surgeries. We depicted one such example in Figure 6 .

Before we dwell into the details we briefly explain the ideas used in the proof of the main theorem: Given a contact $( \pm 1)$-surgery diagram, apply the algorithm in [AO] to embed the surgery curves onto the Seifert surface of a torus knot such that the surface framing of any surgery curve agrees with its contact framing. Here we also allow contact $\frac{1}{k}$-surgeries since we can convert them into a sequence of contact $(+1)$-surgeries. By a slight generalization of the main theorem in $[\mathrm{AO}]$ we get an achiral Lefschetz fibration over $D^{2}$ with bounded fibers which induces an open book 


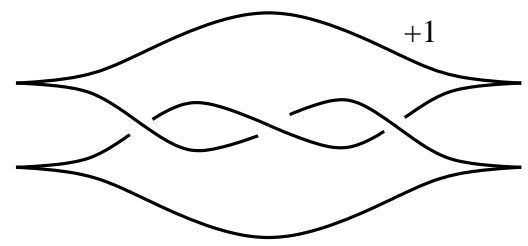

Figure 6. A tight non-fillable contact structure

decomposition on the boundary. In turns out that (cf. [P] and [Sti]) the contact structure given by the surgery diagram is compatible with the induced open book. The page of the open book is the Seifert surface of an appropriate torus knot (embedded in certain way in $S^{3}$ ) and the monodromy is the composition of the monodromy of the torus knot with Dehn twists along the surgery curves embedded in the Seifert surface. A (+1)-surgery curve induces a left-handed Dehn twist while a (-1)-surgery curve induces a right-handed Dehn twist. Moreover the monodromy of the torus knot can be given explicitly as a product of right-handed Dehn twists along certain curves. In short, the algorithm in [AO] for finding a Lefschetz fibration on a Stein surface yields an algorithm for finding an explicit open book compatible with a given contact surgery diagram. The proof is completed by using a criterion given by Goodman ([Go]) for detecting overtwistedness of a contact structure by finding a sobering arc on a page of a compatible open book. We advise the reader to turn to [OS] for the relevant notions.

Acknowledgement: We would like to thank András I. Stipsicz for helpful and inspiring correspondence. The author was partially supported by the Turkish Academy of Sciences.

\section{Monodromy of A TORUS KNOT}

Suppose that for a link $L$ in a 3-manifold $Y$ the complement $Y-L$ admits a locally trivial fibration $f: Y-L \rightarrow S^{1}$ such that the fibers are interiors of Seifert surfaces of $L$. Then $(L, f)$ is called an open book decomposition of $Y$. The Seifert surface $F=\overline{f^{-1}(t)}$ is called the page, while $L$ the binding of the open book decomposition. Any locally trivial bundle with fiber $F$ over an oriented circle is equivalent to the fibration

$$
\frac{I \times F}{(1, x) \sim(0, h(x))} \rightarrow \frac{I}{\partial I} \approx S^{1}
$$

for some self-diffeomorphism $h$ of $F$. The isotopy class represented by the map $h$ is called the monodromy of the fibration, which in turn is called the monodromy of the open book decomposition. We will make no distinction between an open book decomposition and a fibered link. A fibered link is a link $L \subset Y$ whose complement 
$Y-L$ fibers over $S^{1}$, in such a way that each fiber intersects a tubular neighborhood of $L$ in a curve isotopic to $L$. It is clear that the binding of an open book decomposition is a fibered link and conversely a fibered link naturally induces an open book decomposition.

Let $p$ and $q$ be relatively prime integers such that $p, q \geq 2$. It is well-known that a $(p, q)$-torus knot is a fibered knot. This gives an open book decomposition of $S^{3}$, where the fiber is a surface of genus $\frac{1}{2}(p-1)(q-1)$ with one boundary component and the monodromy is a product of $(p-1)(q-1)$ right-handed Dehn twists along non-separating (i.e., homologically non-trivial) curves. Even though the monodromy of a torus knot is discussed extensively in [AO] we repeat this construction here for the sake of completeness.

First we describe the plumbing operation and explain how to construct the fiber of a torus knot by plumbing positive Hopf bands. The monodromy of a positive (resp. negative) Hopf band is a right-handed (resp. left-handed) Dehn twist along its core circle.

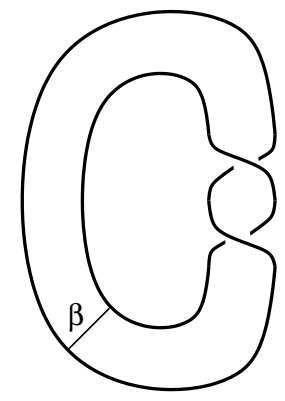

(a)

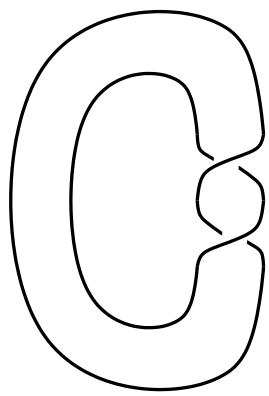

(b)

Figure 7. Positive (a) and negative (b) Hopf bands

Let $H_{+}$denote the positive Hopf link and $F_{+}$its fibered surface (the positive Hopf band) in $S^{3}$ which is shown in Figure 7(a). Suppose that $(L, F)$ is another fibered link with its fibered surface in an arbitrary 3-manifold $Y$. Choose an arc $\alpha$ in $F$ connecting two points on the boundary $L=\partial F$. Take a neighborhood $\nu \alpha$ of $\alpha$ in $F$ and thicken this into a 3-ball. Now apply the same for the curve $\beta$ on the Hopf band $F_{+}$depicted in Figure 7(a) and take a connected sum of $Y$ with $S^{3}$ along these 3-balls such that $\nu \alpha$ and $\nu \beta$ are glued in a way that $\alpha$ is transverse to $\beta$. It is proven in [Sta] (see also [Ga]) that by plumbing two fibered links we get a new fibered link and the monodromy of the new fibered link will be the product of monodromies of the fibered links with which we started. Here to make sense of this product we need to extend 
each of the monodromy homeomorphisms of the fibered surfaces that we are plumbing by identity onto the whole glued up surface so that we have two homeomorphisms to compose both of which are defined on the plumbed surface.

For example we can plumb two positive Hopf links to get a $(2,3)$-torus knot (the right-handed trefoil) with its fibered surface. Simply identify a neighborhood of the arc $\alpha$ in one Hopf band with a neighborhood of the arc $\beta$ in the other Hopf band, transversally as shown in Figure 8(a) and (b). Then move the left leg of the Hopf band in the back over the right leg of the Hopf band in the front to arrive at Figure 8(c). The resulting monodromy will be the product of two right-handed Dehn twists along the curves also drawn in Figure 8(c). Notice that the two monodromy curves intersect each other only once and they stay parallel when they go through the left twist on the surface. We can iterate this plumbing operation to express the monodromy of a $(2, q)$-torus knot as a product of $(q-1)$ right-handed Dehn twists.

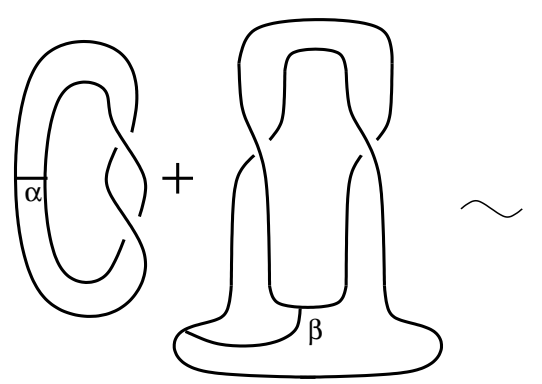

(a)

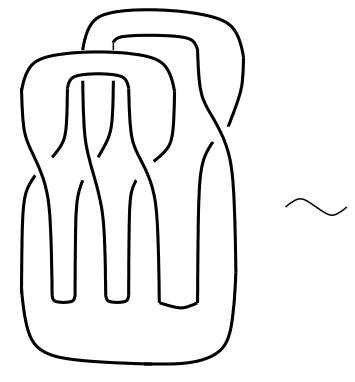

(b)

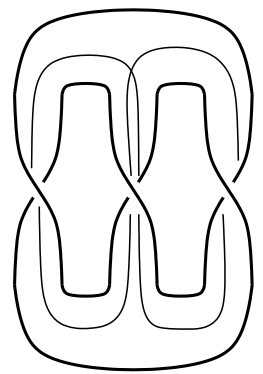

(c)

Figure 8. Plumbing two Hopf bands

By attaching more positive Hopf bands we can construct the fibered surface of a $(p, q)$-torus knot for arbitrary $p$ and $q$. We depicted the $(3,4)$-torus knot with its fibered surface in Figure 9. We can view this figure as two rows of "archways". First construct the row of archways in the back as described above and then plumb a Hopf band in the front row and proceed as above to obtain a second row of archways. We can iterate this process to build as many rows of arcways as we wish. Hence the monodromy of a torus knot is a product of right-handed Dehn twists along certain curves explicitly determined by this process. The numbers associated to the monodromy curves in Figure 9 indicate the order we should follow in writing the monodromy as a product of right-handed Dehn twists along these curves.

Next we briefly describe the Seifert surface of a torus knot by a different construction. We can obtain the Seifert surface of a $(p, q)$-torus knot starting with $p$ horizontal 


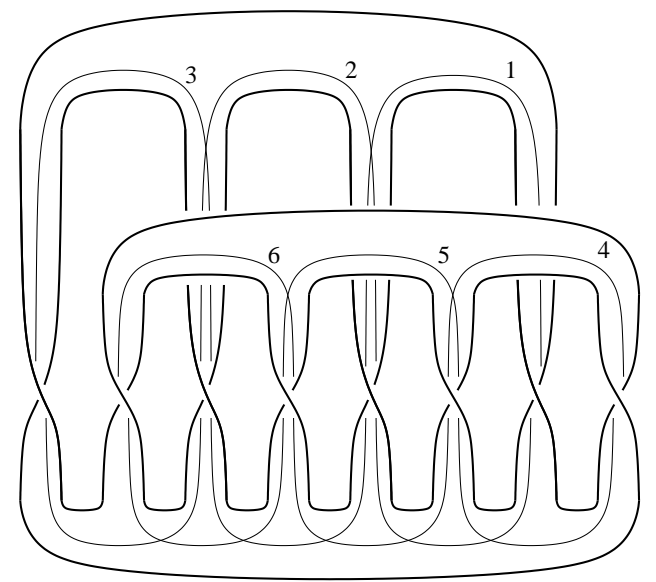

Figure 9. Monodromy of a $(3,4)$ torus knot

and $q$ vertical strips (i.e., disks) and by connecting them with small bands. In Figure 10 we depicted the Seifert surface of a $(3,4)$-torus knot and the monodromy curves on it. Again the numbers associated to the curves in Figure 10 denote the order we should compose these curves when writing the monodromy as a product. The embeddings of the surfaces depicted in Figure 9 and Figure 10 are of course isotopic. To see this isotopy, visualize pulling down the horizontal front strip in Figure 10, flipping it over and then pushing the other horizontal strips (except the top one which is all the way in the back) slightly to the right.

\section{An EXPLICIT OPEN BOOK DECOMPOSITION COMPATIBLE WITH A GIVEN CONTACT STRUCTURE}

Recently Giroux proved a central result about the topology of contact 3-manifolds. He established a one-to-one correspondence between contact structures (up to isotopy) and open book decompositions (up to positive stabilization/destabilization) on a closed oriented 3-manifold. In this section we describe an explicit construction of an open book decomposition compatible with a given contact structure.

We start with a contact $( \pm 1)$-surgery diagram and then rotate this diagram to put it in a square bridge position as illustrated in Figure 11 for the right-handed trefoil knot. Now first we assume that all the curves in the diagram have $( \pm 1)$ surgery coefficients. We will modify the construction later in the presence of contact $\frac{1}{k}$-surgeries, for $k>0$. Once put in a square bridge position any part of the link will be in either a horizontal or a vertical position and the horizontal arcs will go over the 


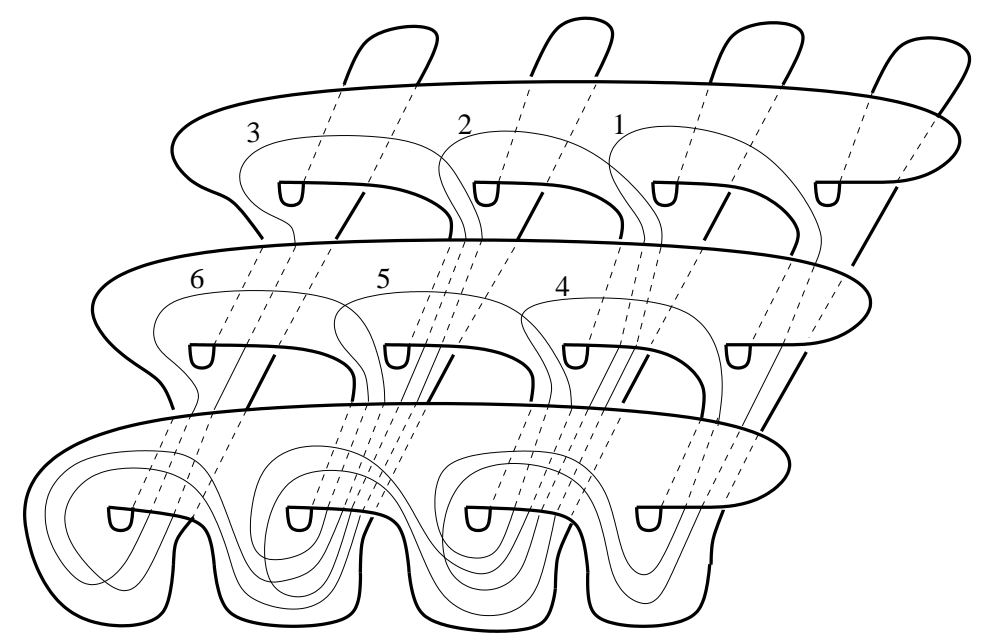

Figure 10. Monodromy of a $(3,4)$-torus knot; a different view

vertical ones. We can assume that on each horizontal and vertical line there is only a connected piece of the given link.
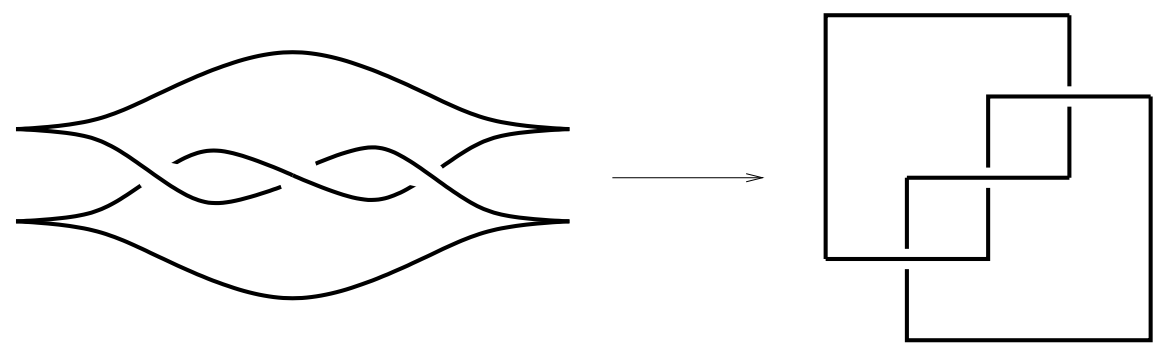

FiguRE 11. Rotating a Legendrian link to put into square bridge position

Next we repeat the construction of a torus knot as described at the end of Section 1 and embed the given Legendrian link onto the Seifert surface of the appropriate torus knot. In Figure 12 we illustrated this process for the unknot depicted in Figure 3. Although it would be sufficient to use the Seifert surface of a $(3,3)$-torus link we preferred to use a $(3,4)$-torus knot (by adding the right-most dashed vertical line in Figure 12) to have a connected boundary which is not very essential.

Now we have an open book decomposition whose page is the Seifert surface of the torus knot and whose monodromy is the composition of the monodromy of the torus knot and Dehn twists along the components of the surgery link. Here all the Dehn twists of the monodromy of the torus knot is right-handed while a $(+1)$-surgery curve 


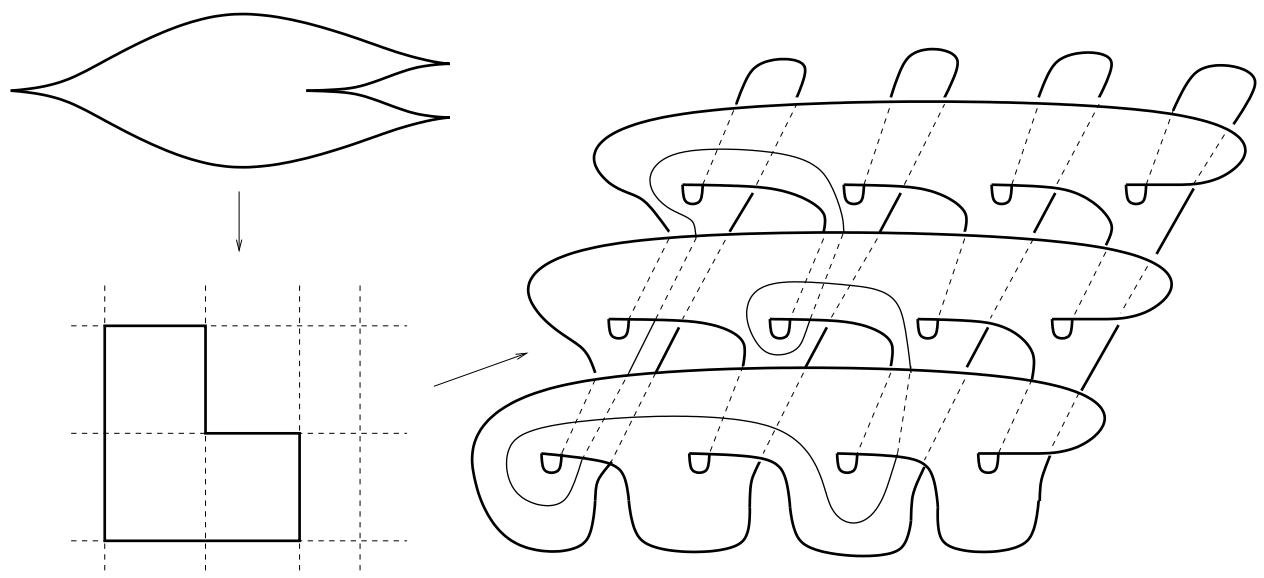

FiguRE 12. Embedding a Legendrian link onto the Seifert surface

(resp. (-1)) induces a left-handed (resp. right-handed) Dehn twist. Notice that the surgery curves are pairwise disjoint and they are homologically non-trivial on the Seifert surface by this construction. By $[\mathrm{P}]$ in the case of only $(-1)$-surgery curves and by [Sti] in the general case this open book decomposition is compatible with the contact structure induced by the given contact surgery diagram.

Finally assume that there is a surgery curve $K$ with $\frac{1}{k}$-coefficient in the given surgery diagram. We apply the same algorithm as above to embed all the curves onto the Seifert surface of an appropriate torus knot (without replacing the knot $K$ with its $k$ successive Legendrian push-offs). Then we observe that since contact $\frac{1}{k}$-surgery along $K$ is equivalent to a sequence of contact $(+1)$-surgeries on the $k$ successive Legendrian push-offs of $K$, performing a contact $\frac{1}{k}$-surgery will correspond to a product of left-handed Dehn twists along $k$ parallel copies of the embedded curve $K$ on the Seifert surface. Here notice that the Legendrian push-offs of $K$ will stay on the Seifert surface since the contact framing of $K$ agrees with its surface framing. In other words, in the presence of a contact $\frac{1}{k}$-surgery curve $K$ in the diagram we modify our algorithm above by taking the $k$-th power of the left-handed Dehn twist corresponding to the surgery curve $K$.

\section{GOODMAN'S CRITERION FOR OVERTWISTEDNESS}

In this section we will discuss a criterion given by Goodman ([Go]) to detect overtwistedness of a contact structure by examining the monodromy of a compatible open book decomposition. Notice that the contact structures compatible with a given open book decomposition are all tight or all overtwisted. (The reason is that all contact structures compatible with a given open book are isotopic.) Hence we will call an open 
book decomposition overtwisted if a contact structure compatible with this open book decomposition is overtwisted. Let $\alpha, \beta \subset F$ be properly embedded oriented arcs which intersects transversely on an oriented surface $F$. The algebraic intersection number $i_{a l g}(\alpha, \beta)$ is the oriented sum over interior intersections. The geometric intersection number $i_{\text {geom }}(\alpha, \beta)$ is the unassigned count of interior intersections, minimized over all boundary fixing isotopies of $\alpha$ and $\beta$. The boundary intersection number $i_{\partial}(\alpha, \beta)$ is one-half the oriented sum over intersections at the boundaries of the arcs, after the arcs have been isotoped, fixing boundary, to minimize geometric intersection. (See Figure 13 for sign conventions.)
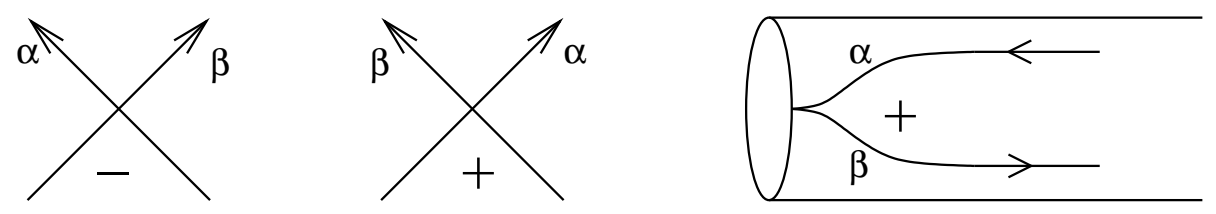

FiguRE 13. Sign convention for intersection numbers on a surface

In particular, given an arc $\alpha$ on a page $F$ of an open book decomposition with monodromy $h$, we can consider $i_{a l g}(\alpha, h(\alpha)), i_{\text {geom }}(\alpha, h(\alpha))$ and $i_{\partial}(\alpha, h(\alpha))$. Here $h(\alpha)$ is oriented by reversing the orientation on $h(\alpha)$ which is obtained by pushingforward the orientation of $\alpha$ by the monodromy $h$.

Definition 1. A properly embedded arc $\alpha$ is sobering for a monodromy $h$, if

$$
i_{\text {alg }}(\alpha, h(\alpha))+i_{\text {geom }}(\alpha, h(\alpha))+i_{\partial}(\alpha, h(\alpha)) \leq 0,
$$

and $\alpha$ is not isotopic to $h(\alpha)$.

The following result is due to Goodman.

Proposition 2. If there is a sobering arc $\alpha \subset F$ for $h$, then the given open book decomposition is overtwisted.

In order to prove this result, Goodman constructs a surface with Legendrian boundary which violates Eliasberg's well-known inequality (cf. [E]) for tight contact 3manifolds. We note that Goodman's criterion does not explicitly produce an overtwisted disk.

\section{Main Theorem}

Theorem 3. Given a contact surgery diagram which contains a $\frac{1}{k}$-surgery curve for some positive integer $k$. Then the contact structure obtained by stabilizing (cf. Figure 2) an isolated arc of the $\frac{1}{k}$-surgery curve is overtwisted. 
Proof. Given a contact surgery diagram which contains a $\frac{1}{k}$-surgery curve $K$. First we apply the algorithm in Section 2 to find an explicit open book decomposition compatible with the contact structure induced by the given diagram. Then we consider the surgery curve $K$ that we are going to stabilize which is embedded in an appropriate Seifert surface obtained by the above algorithm. Now we can isotope the isolated part of this curve on the surface to move it near the boundary of the surface. Next we stabilize our open book decomposition by attaching a positive Hopf band in a small part of the boundary and define a new curve $K^{\prime}$ which is a connected sum of $K$ with the core circle $C$ of the attached Hopf band as depicted in Figure 14.

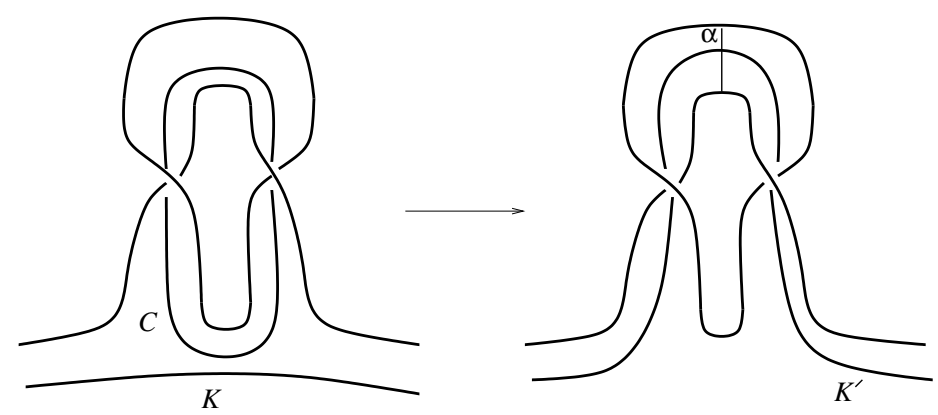

FiguRE 14. The sobering $\operatorname{arc} \alpha$

So we get a new open book decomposition compatible with the contact structure (cf. [Et]) induced by the surgery diagram in which we replace $K$ by its once stabilized Legendrian copy $K^{\prime}$. The page of the new open book will have an extra 1-handle and we will twist along $K^{\prime}$ (which runs over this new 1-handle once) instead of $K$ in the new monodromy.

We will prove that this contact structure is overtwisted by showing that the arc $\alpha$ across the attached Hopf band in Figure 14 is a sobering arc for the new open book decomposition. Notice that the contribution of this positive Hopf band to the monodromy $h$ of the new open book is a right-handed Dehn twist along $C$ which is disjoint from all the other curves in the monodromy. To find the image $h(\alpha)$ we only need to consider a right-handed Dehn twist along $C$ and $k$ consecutive left-handed Dehn twists along $K^{\prime}$ since the other monodromy curves will have no affect on the image of $\alpha$. In Figure 15 we depict the monodromy curves intersecting $\alpha$ and in Figure 16 we show the image of $\alpha$ under the monodromy $h$.

We can check that $\alpha$ is a sobering arc since the sign of each interior intersection of $\alpha$ and $h(\alpha)$ is negative and the contributions from the boundary intersections cancel out (see Figure 17) with the given sign conventions in Figure 13. Recall that we need to reverse the orientation on $h(\alpha)$ induced by pushing-forward any fixed orientation 


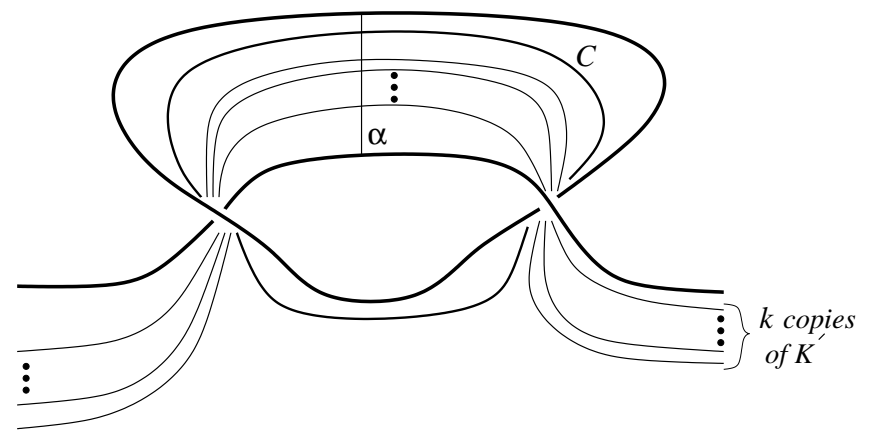

FiguRE 15. Monodromy curves intersecting $\alpha$

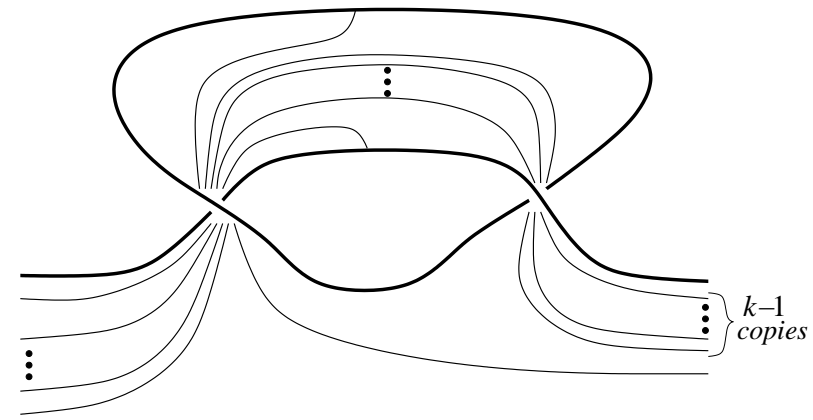

FIGURE 16. Image of $\alpha$ under the monodromy

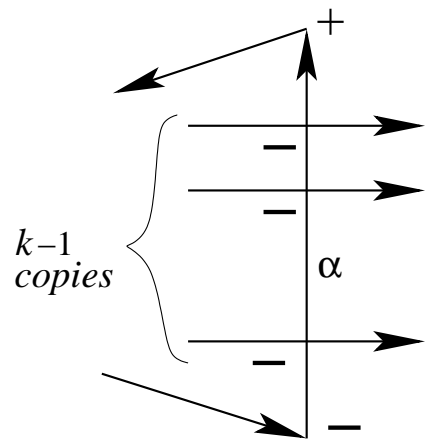

FiguRE 17. Intersection points of $\alpha$ and its image under the monodromy 
on $\alpha$. Thus the result follows from Goodman's criterion (cf. Proposition 2) since we showed that

$$
i_{\text {alg }}(\alpha, h(\alpha))+i_{\text {geom }}(\alpha, h(\alpha))+i_{\partial}(\alpha, h(\alpha))=-(k-1)+(k-1)+0=0 .
$$

Notice also that $\alpha$ is clearly not isotopic to its image $h(\alpha)$.

As an example consider a contact $\frac{1}{k}$-surgery on the Legendrian unknot of Figure 3. The result is an overtwisted contact structure for any positive integer $k$.

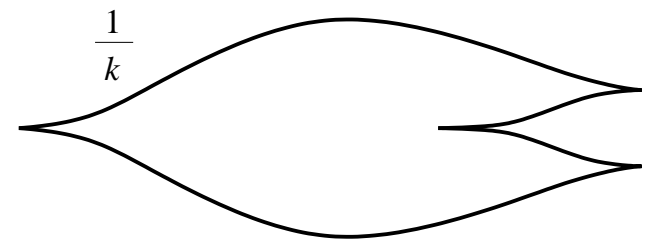

FigURE 18. A family of overtwisted contact structures

\section{SOME REMARKS}

In Figure 1 we gave an example of a contact structure which is tight. If we apply the same algorithm to this diagram (without stabilizing it) we will not be able to find a sobering arc - as expected. If we try the arc $\alpha$ we picked for the proof above, either $\alpha$ will be isotopic to its image under the monodromy $h$ of the open book or we will have

$$
i_{\text {alg }}(\alpha, h(\alpha))+i_{\text {geom }}(\alpha, h(\alpha))+i_{\partial}(\alpha, h(\alpha))=i_{\partial}(\alpha, h(\alpha))=+1 .
$$

In fact we can see that this contact structure on $S^{1} \times S^{2}$ is tight as follows: Rotate the unknot to put it in square bridge position (which is just a square in this case) and embed it on the Seifert surface of a $(3,4)$-torus knot. Actually we only need the Seifert surface of the $(2,2)$-torus link (i.e., an annulus) but we picked the $(3,4)$-torus knot to show the flexibility of the construction. Without loss of generality we can assume that this $(+1)$-surgery curve is embedded as the 6th curve in Figure 10. So the total monodromy of the open book compatible with the contact surgery will be the product of five right-handed Dehn twist corresponding to the first five curves in Figure 10 since the last right-handed Dehn twist corresponding to the 6th curve will be cancelled out by the left-handed Dehn twist corresponding to the $(+1)$-surgery curve. Thus the contact structure at hand is Stein fillable (by a theorem of Giroux) and hence tight. 
On the other hand, if we perform a contact $\frac{1}{k}$-surgery on the unknot of Figure 1 we can easily see by using the techniques in this note that the resulting contact structure is overtwisted for all $k>1$.

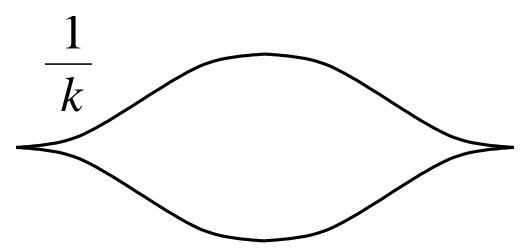

FiguRE 19. Overtwisted contact structures for $k \geq 2$

This last example becomes interesting when compared with the case of contact $\frac{1}{k}$-surgery on the Legendrian trefoil knot which is tight for all $k \geq 1$ (cf. [LS]).

\section{REFERENCES}

[AO] S. Akbulut and B. Ozbagci, Lefschetz fibrations on compact Stein surfaces, Geom. Topol. 5 (2001), 319-334.

[DG] F. Ding and H. Geiges, A Legendrian surgery presentation of contact 3-manifolds, Proc. Cambridge. Philos. Soc. 136 (2004), 583-598.

[DGS1] F. Ding, H. Geiges and A. Stipsicz, Surgery diagrams for contact 3-manifolds, Proceedings of the Gökova Geometry-Topology Conference (2003), arXiv:math.SG/0307237

[DGS2] F. Ding, H. Geiges and A. Stipsicz, Lutz twist and contact surgery, preprint, arXiv:math.SG/0401338

[E] Y. Eliashberg, Contact 3-manifolds twenty years since J. Martinet's work, Ann. Inst. Fourier, 42 (1992), 165-192.

[Et] J. Etnyre, Planar open book decompositions and contact structures, preprint, arXiv:math.SG/0404267

[Ga] D. Gabai, Detecting fibered links in $S^{3}$, Comment. Math. Helv. 61 (1986), 519-555.

[Go] N. Goodman, Contact structures and open books, Ph.D thesis, UT Austin, 2003.

[LS] P. Lisca and A. Stipsicz, Seifert fibered contact 3-manifolds via surgery, Alg. Geom. Top. 4 (2004), 199-217.

[OS] B. Ozbagci and A. Stipsicz, Surgery on contact 3-manifolds and Stein surfaces, Bolyai Society Mathematical Studies, to appear.

[P] O. Plamenevskaya, Contact structures with distinct Heegaard-Floer invariants, preprint, arXiv:math.SG/0309326

[Sta] J. Stallings, Construction of fibered knots and links, A.M.S. Proc. Symp. in Pure Math. 32 (1978), 55-60.

[Sti] A. Stipsicz, Surgery diagrams and open book decompositions, to appear in Acta Math. Hung., 2003.

Department of Mathematics, Koç University, Istanbul, Turkey

E-mail address: bozbagci@ku.edu.tr 